\title{
Nuevos datos del uso de la costa en el extremo sur del continente por cazadores-recolectores durante el Holoceno tardío. El sitio Cabo Vírgenes 24 (Santa Cruz, Argentina)
}

RMA

Arqueología

\author{
New data on hunter gatherer coastal use at the southern \\ tip of the continent during the late Holocene. Cabo \\ Virgenes 24 site (Santa Cruz, Argentina)
}

Flavia Carballo Marina*, Juan Bautista Belardi** y G. Lorena L'Heureux***

*Universidad Nacional de la Patagonia Austral (UARG-ICASUR), E-mail: flaviacarballomarina@gmail.com; ${ }^{* *}$ CONICET y Universidad Nacional de la Patagonia Austral (UARG-ICASUR), E-mail: juanbautistabelardi@gmail.com;

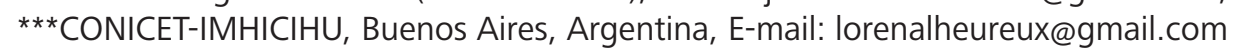

\begin{abstract}
Resumen
El sitio Cabo Vírgenes 24 (CV24) se ubica en el extremo sureste de la Patagonia continental y presenta ocupaciones entre 753-888 (1270 \pm 60 14C A.P - LP3229) y 545-626 años Cal A.P (619 26 14C A.P - AA106800). Los artefactos líticos se confeccionaron con materias primas disponibles localmente (dacita y RGFO). El componente expeditivo de la tecnología concuerda con la de cazadores de guanacos del interior del continente. Se observa una baja densidad y riqueza moderada de especies marinas y terrestres con un énfasis en la explotación de pinnípedos (Arctocephalus australis y Otaria flavescens), seguida de guanacos (Lama guanicoe) y un menor uso de aves marinas (Phalacrocorax sp., Spheniscus magellanicus y Aptenodytes patagonicus). La información provista por CV24 reafirma las tendencias observadas respecto de la utilización esporádica y marginal de la localidad de Cabo Vírgenes por parte de cazadoresrecolectores desde el Holoceno tardío hasta el contacto con los europeos.
\end{abstract}

Palabras claves: Holoceno tardío; costa patagónica; cazadores-recolectores del interior; tecnología expeditiva; explotación de recursos terrestres y marinos.

\begin{abstract}
Cabo Virgenes 24 (CV24) is an archaeological site located at the Southeastern end of continental Patagonia and

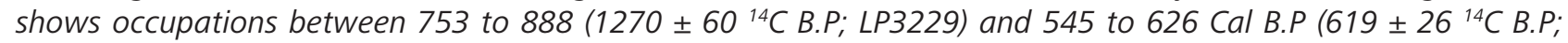
AA106800). Lithic artifacts were mainly made on locally available raw material (dacite and RGFO). The identified technological expedient component is in accordance with inland guanaco hunters. The faunal record shows low density and moderate richness of marine and terrestrial faunal species. There is an emphasis on exploitation of pinnipeds (Arctocephalus australis and Otaria flavescens), followed by guanaco (Lama guanicoe) and, to a lesser extent, seabirds (Phalacrocorax sp., Spheniscus magellanicus and Aptenodytes patagonicus). The archaeological record of CV24 reaffirms the observed trends related to the sporadic and marginal use of the Cabo Vírgenes locality by hunter-gatherer populations since the Late Holocene until the European contact.
\end{abstract}

Key words: Late Holocene; coastal Patagonia; inland hunter-gatherer; expedient technology; marine and terrestrial resources exploitation.

\section{CV24 y antecedentes regionales}

El sitio Cabo Vírgenes 24 (CV24) se ubica en el extremo sureste de la Patagonia continental argentina $\left(52^{\circ}\right.$ $\left.19^{\prime} \mathrm{S} ; 68^{\circ} 22^{\prime} \mathrm{O}\right)$, dentro de la Reserva Provincial Cabo Vírgenes, a $11 \mathrm{msnm}$ (Figura 1.A). Se localiza al pie del palaeoacantilado formado por depósitos activos de drift, sobre la playa de erosión del océano Atlántico. La formación de este espacio se inició en el Holoceno medio o tardío (González Bonorino 2002, entre otros).

Como resultado de investigaciones previas se reconocieron sitios costeros (eg. CV1, CV2, CV6, CV8, CV17) que proveyeron abundante evidencia arqueológica, geológica y tafonómica (Borrero y Franco 2005; Borrero et al. 2008). Estos estudios demostraron que los cazadores-recolectores del interior comenzaron a utilizar este espacio de forma marginal desde los 2000 años A.P. Con posterioridad, producto del impacto por obras antrópicas se identificó 


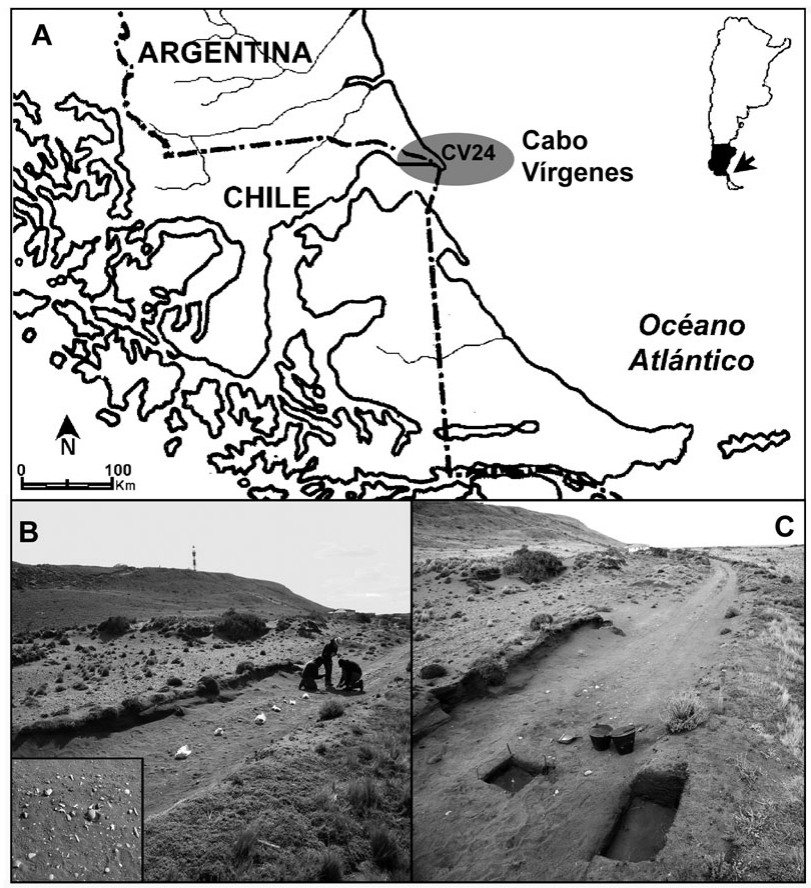

el sitio CV20 (Belardi et al. 2011; L'Heureux et al. 2012). En el año 2014 la erosión eólica sobre un camino interno expuso el sitio CV24. El mismo fue impactado de forma directa por la circulación de vehículos que dispersó materiales líticos y óseos (Figura 1.B y C). Se los recuperó (Figura 1.B) empleando 15 unidades de recolección contiguas de un metro de largo por el ancho del camino 3,2 m. A la vez, se realizaron dos sondeos de 0,5 $\mathrm{m}^{2}$ en sectores no impactados al costado del mismo. Los materiales provenían de un único nivel ubicado entre 28 y $48 \mathrm{~cm}$ de profundidad. No se identificó el paleosuelo reconocido en el área datado sobre la base de fechados OCR en torno al siglo X AD (entre otros, Barberena y Borrero 2010). También se recolectaron artefactos líticos en menor frecuencia sobre un segundo camino que conecta con el sitio CV20. Tanto los materiales de superficie como los de estratigrafía fueron analizados

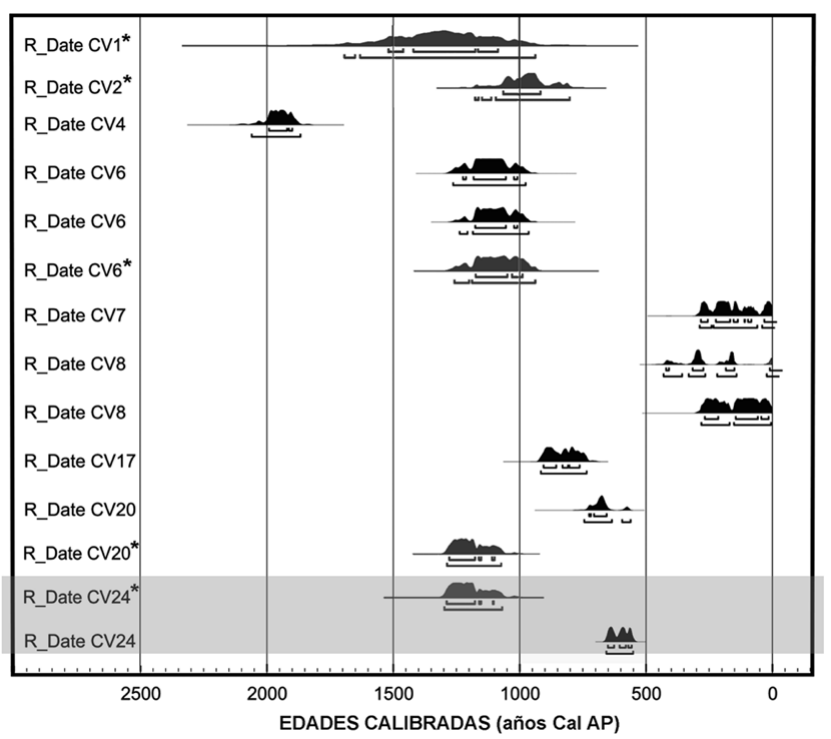

Figura 1. A: Área de Cabo Vírgenes y localización del sitio CV24. B y C: Vista de la dispersión de materiales arqueológicos sobre el camino y recuperados a partir de unidades de recolección y sondeos.

Figure 1. A: Cabo Vírgenes area and CV24 site location. B and C: Archaeological materials dispersion view and the road and materials recovered from the recollection and excavated units.

como parte de un mismo conjunto.

Se obtuvieron dos fechados radiocarbónicos de muestras de superficie, uno sobre un fémur de lobo marino (Pinnipedia) -edad corregida por efecto reservorio- de 870 \pm 70 años ${ }^{14}$ C A.P, LP 3229 (753-888 años Cal A.P), y otro sobre un húmero distal de guanaco (Lama guanicoe) de $619 \pm 26$ años ${ }^{14} \mathrm{C}$ A.P ( $\delta 13^{\mathrm{C}} \%$ o -20.8), AA106800 (545626 años Cal A.P). En la figura 2 se muestran curvas de dataciones para Cabo Vírgenes generadas sobre la base de los fechados radiocarbónicos calibrados de CV24 y de otros sitios del área (1 $\sigma)$.

\section{Evidencia lítica en CV24}

Los artefactos líticos ( $\mathrm{N}=98$; Tabla 1) fueron confeccionados principalmente con rocas disponibles localmente (en el drift glacial sobre el paleoacantilado y en los cordones litorales): dacita $(63,26 \%)$ y RGFO (rocas de grano fino oscuras sensu Charlin 2005) (33,6\%). Las mismas rocas fueron empleadas en los artefactos reconocidos en los alrededores del sitio ( $\mathrm{N}=23$; Tabla 1), a lo largo del segundo camino y en cercanías de CV20 (ver Belardi et al. 2011). Además, la presencia de corteza y de talones lisos y corticales (87\%) -que indican la no preparación de núcleos para la talla-, sugieren un importante componente expeditivo que se ajusta a la tecnología de los cazadores de guanaco del interior. Esto, junto con el conjunto de artefactos formatizados en el que predominan las raederas, es concordante con lo conocido en el área (Figura 3).

\section{La explotación de la fauna}

Se recuperaron 369 especímenes correspondientes a cinco taxa de Mammalia (NISP 141), cuatro de Aves (NISP 72) y tres de Mollusca (NISP 156) (Tabla 2). Los análisis de diversidad exponen una baja densidad en relación con la superficie excavada, y una riqueza moderada de especies, tanto de fauna marina como terrestre (Figura 4.A). Se registra un énfasis en la explotación de pinnípedos (Arctocephalus australis y/o Otaria flavescens, 18,43\%), seguidos de mamíferos terrestres (Lama guanicoe, 8,4\%). A diferencia de otros sitios del área (ver Borrero et al. 2008), las aves marinas no representan el principal recurso (Phalacrocorax sp. -4,61\%-; Spheniscus magellanicus y Aptenodytes patagónicus -6,78\%) (Tabla 2).

Figura 2: Curva de dataciones calibradas de los fechados radiocarbónicos de Cabo Vírgenes (1 $\sigma$; OxCal v4.2.4). *Edades corregidas por efecto reservorio.

Figure 2: Curve of calibrated dates of the radiocarbon dating of Cabo Vírgenes (1 $\sigma$; OxCal v4.2.4). * Ages corrected by reservoir effect. 


\begin{tabular}{|c|c|c|c|c|c|}
\hline \multirow{2}{*}{$\begin{array}{c}\text { CV24 } \\
\text { Tipo de artefacto }\end{array}$} & \multicolumn{5}{|c|}{ Materias primas } \\
\hline & RGFO & Dacita & Granito & Calcedonia & $\begin{array}{c}\text { TOTAL y } \\
(\%)\end{array}$ \\
\hline Lasca cortical & 5 & 9 & 0 & 1 & $15(15,30)$ \\
\hline Lasca interna & 24 & 45 & 0 & 1 & $70(71,42)$ \\
\hline Núcleo & 2 & 2 & 0 & 0 & $4(4,08)$ \\
\hline Raedera & 1 & 3 & 0 & 0 & $4(4,08)$ \\
\hline Biface & 1 & 1 & 0 & 0 & $2(2,04)$ \\
\hline Punta de proyectil & 0 & 1 & 0 & 0 & $1(1,02)$ \\
\hline $\begin{array}{l}\text { Fragmento de } \\
\text { artefacto formalizado }\end{array}$ & 0 & 1 & 0 & 0 & $1(1,02)$ \\
\hline Bola & 0 & 0 & 1 & 0 & $1(1,02)$ \\
\hline TOTAL y (\%) & $33(33,6)$ & $62(63,26)$ & $1(1,02)$ & $2(2,04)$ & $98(100)$ \\
\hline \multicolumn{2}{|c|}{ Alrededores del sitio CV 24} & \multicolumn{4}{|c|}{ Materias primas } \\
\hline \multicolumn{2}{|c|}{ Tipo de artefacto } & \multicolumn{2}{|c|}{ RGFO } & Dacita & TOTAL \\
\hline Lasca cortical & & 8 & 0 & 8 & \\
\hline Lasca internas & & 9 & 0 & 9 & \\
\hline Raedera & & 1 & 0 & 1 & \\
\hline Raspador & & 0 & 1 & & \\
\hline Cuchillo & & 0 & 1 & & \\
\hline Punta de proyectil & & 1 & 0 & & \\
\hline Fragmento de artefactc & formatizado & 1 & 1 & 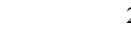 & \\
\hline TOTAL & & 20 & 3 & & 3 \\
\hline
\end{tabular}

Tabla 1: Clases de artefactos y materias primas presentes en CV24 (además, se registraron 85 desechos de talla sin talón -42 de basalto, 41 de dacita y dos de calcedonia) y en los alrededores de CV24 (además, hay 20 desechos de talla sin talón -19 de basalto y uno de sílice).

Table 1: Classes of artifacts and raw materials present in CV24 (in addition, 85 wastes of heap size were recorded -42 of basalt, 41 of dacite and two of chalcedony) and in the vicinity of CV24 (in addition, 20 wastes of Size without heel -19 of basalt and one of silica).

El perfil de meteorización (Behrensmeyer 1978) del material de superficie difiere del de excavación. Es más visible en los pinnípedos, con un perfil bajo-moderado en estratigrafía con predominio del estadio 1 (40\%), y moderado-alto en superficie con mayoría del 3 (51,72\%). Los restos de guanaco tienen un perfil moderado-alto en superficie, con predominio del estadio 2 (37\%) y del 3 $(29,63 \%)$ y bajo-moderado en estratigrafía con mayoría de estadios 1 y 2 (25 y 75\% respectivamente). En ambos taxa se dan casos de estadios avanzados -4- en superficie, ausentes en el material de excavación.

Las marcas de procesamiento ocurren con mayor frecuencia sobre los huesos de cormoranes y pinnípedos mientras que las fracturas transversales y longitudinales en los huesos largos de guanaco (Figura 4.B y C). Lo señalado y la frecuencia de individuos explotados por taxa, muestran la preferencia por la fauna marina de elevados niveles tróficos.

\section{CV24 en el marco arqueológico regional}

El hiato entre las fechas radiocarbónicas y la amplia

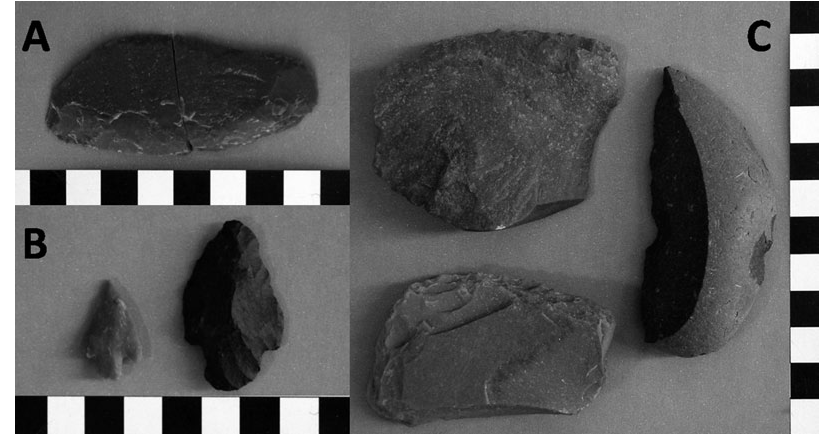

Figura 3: A: Cuchillo; B: puntas de proyectil; C: raederas (se observa la corteza en el ejemplar de la derecha y en el inferior).

Figure 3: A: Knife; B: projectile points; C: scrappers (the bark is observed in the specimen on the right and in the lower one).
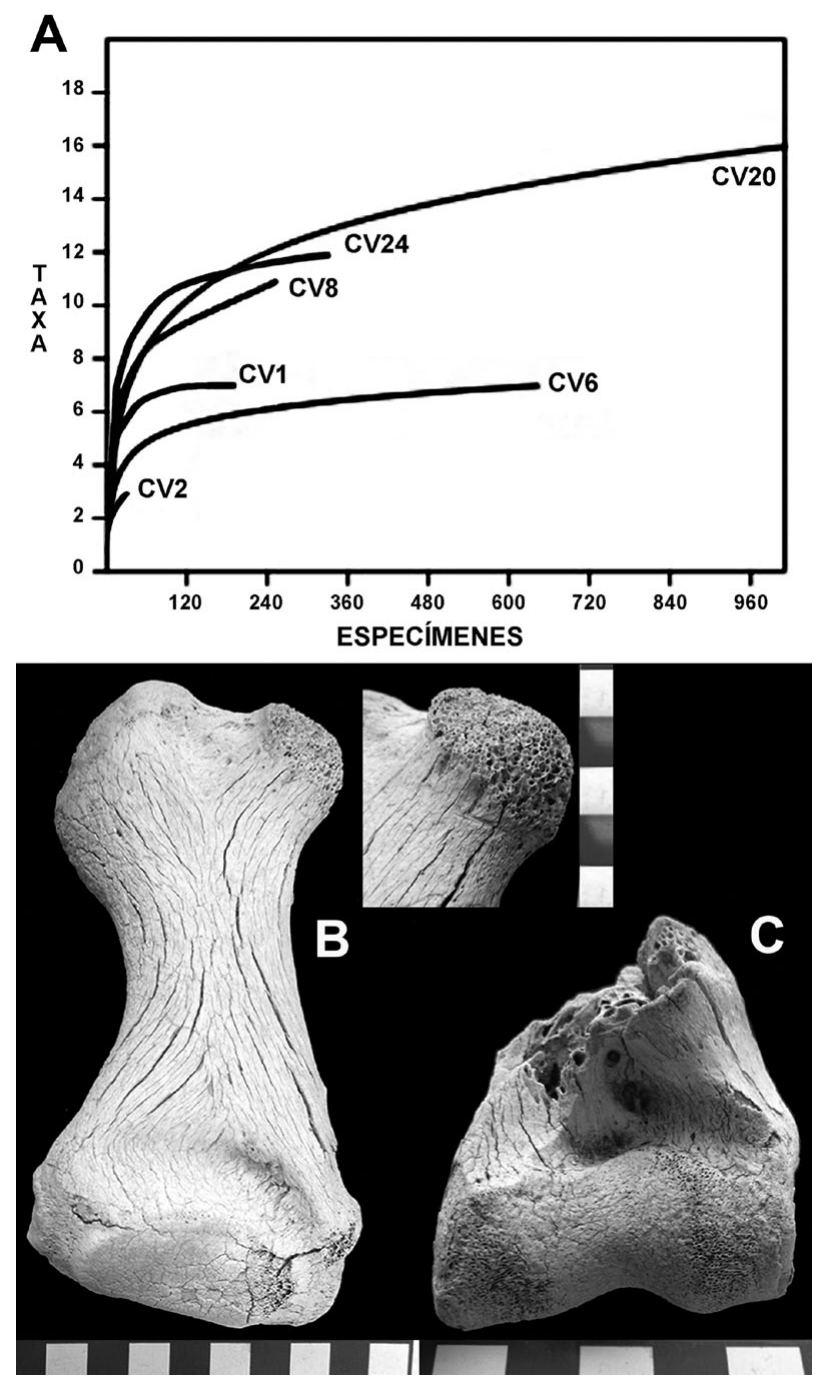

Figura 4: A: Riqueza de especies en sitios de la localidad Cabo Vírgenes. Curvas de Rarefacción; B: Marcas de corte debajo de la cabeza de un fémur derecho de lobo marino; C: fractura transversal en un húmero distal de guanaco. Se observa el alto grado de meteorización de los elementos recuperados en superficie.

Figure 4: A: Species richness in the locality of Cabo Vírgenes sites. Rarefaction curves; B: Cut marks below the head of a right sea lion femur; C: transverse fracture in a distal guanaco humerus. The high degree of weathering of the surface recovered elements is observed. 


\begin{tabular}{lcccc}
\hline MAMMALIA & NISP & MNI & HUELLAS FRACTURAS \\
\hline Lama guanicoe & 31 & 2 & 1 & 4 \\
Ovis aries & 2 & 1 & 0 & 0 \\
Mammalia & 22 & 1 & 0 & 0 \\
Ctenomys sp. & 18 & 2 & 0 & 0 \\
Pinnipedia & 68 & 3 & 3 & 1 \\
Aptenodytes patagonica & 17 & 1 & 2 & 0 \\
Spheniscus magellanicus & 8 & 1 & 0 & 0 \\
Phalacrocorax sp. & 17 & 3 & 3 & 0 \\
Aves & 30 & 2 & 0 & 1 \\
Mytilus sp. & 85 & 37 & -- & -- \\
Aulacomya ater & 4 & 2 & -- & -- \\
Trophon geversianus & 66 & 10 & -- & -- \\
Mollusca & 156 & 50 & -- & -- \\
\hline CV24 TOTAL & $\mathbf{3 6 9}$ & $\mathbf{6 6}$ & $\mathbf{9}$ & $\mathbf{6}$ \\
\hline
\end{tabular}

distribución espacial del material arqueológico a lo largo del camino sugiere que el sitio CV24 es un palimpsesto. Las dataciones coinciden con el momento de mayor actividad humana en Cabo Vírgenes, enfatizando la ocupación recurrente del área a fines del Holoceno tardío hasta el contacto con los europeos.

Se emplearon materias primas líticas de disponibilidad local (dacita y RGFO), y la diversidad y tipos de artefactos se asemejan a los ya registrados en el área, como así también el componente tecnológico expeditivo. El conjunto óseo mostró que la explotación de la fauna se centralizó sobre los mamíferos marinos y el guanaco, cuya representación es relativamente escasa en otros sitios costeros del área. Las frecuencias, la riqueza y la densidad de taxa resaltan que CV24 es un locus con escasa evidencia de uso humano en relación con la brindada por otros sitios de la localidad de Cabo Vírgenes (eg. CV6; CV20).

El sitio CV24 proporciona evidencia nueva y reafirma la utilización esporádica y marginal de Cabo Vírgenes por parte de las poblaciones de cazadores-recolectores del interior, que utilizaron este área de forma discontinua y con menor intensidad que otras zonas aledañas, como la costa Norte del estrecho de Magallanes (Massone 1984; Borrero et al. 2008). Por último, las condiciones de hallazgo de CV24 y de CV20 alertan sobre la vulnerabilidad del registro arqueológico en la localidad de Cabo Vírgenes.

Río Gallegos, 23 de Junio de 2016

\section{Agradecimientos}

A la Dirección de Fauna del Consejo Agrario Provincial (Santa Cruz, Argentina) y a los guardafaunas de la Reserva Provincial Cabo Vírgenes, Sres. Pablo Núñez y Fabián Tejerizo por su colaboración durante los trabajos
Tabla 2: Medidas de abundancia y frecuencias de rastros de actividad humana sobre los restos los óseos de CV24.

Table 2: Measures of abundance and frequencies of traces of human activity on skeletal remains of CV24.

de campo. A Luis Borrero y a dos evaluadores anónimos por sus comentarios.

\section{Bibliografía}

Barberena, R., y L. A. Borrero. 2010. Geoarqueología y distribuciones subsuperficiales de materiales arqueológicos: localidad de Cabo Vírgenes. Editores L. A. Borrero y J. Charlin, Arqueología de Pali Aike y Cabo Vírgenes, Cap. 5 pp: 103-122, CONICET-IMHICIHU, Buenos Aires.

Belardi, J., F. Carballo Marina y G. L. L'Heureux. 2011. Nuevos resultados arqueológicos en Cabo Vírgenes (Santa Cruz, Argentina): el sitio Cabo Vírgenes 20. Magallania 39(2): 279-292.

Behrensmeyer, A. K. 1978. Taphonomic and ecologic information from bone weathering. Paleobiology 4(2): 150-162.

Borrero, L. A., N. V. Franco, R. Barberena, F. Borella, P. Campan, F. Carballo Marina, I. Cruz, C. Favier Dubois, R. A. Guichón, G. L. L'Heureux, M. V. Mancini, L. Manzi, y F. M. Martin. 2008. Arqueología en Cabo Vírgenes y cañadón Gap. Editores I. Cruz y M. S. Caracotche, Arqueología de la costa patagónica. Perspectivas para la conservación, Cap. 13, pp: 212-228. Universidad Nacional de la Patagonia Austral, Río Gallegos.

Charlin, J. 2005. Aprovisionamiento de materias primas líticas en el campo volcánico de Pali Aike (Santa Cruz): una primera aproximación a partir del análisis de los núcleos. Werken 7(2): 39-55.

González Bonorino, G. 2002. Erosión y acreción litoral durante el Holoceno, en especial referencia a las puntas Bustamante y Dungeness. Editor M. J. Haller, Geología y Recursos Naturales de Santa Cruz, 20: 317-324. XV Congreso Geológico Argentino, El Calafate.

L'Heureux, G. L, J. Belardi y F. Carballo Marina 2012. Exploitation of fauna at Cabo Vírgenes, Patagonia Argentina, during the late Holocene. Editor Christine Lefebre, Proceedings of the General Session of the 11th International Council for Archaeozoology Conference, pp. 237-244. BAR 2354, Archaeopress, Oxford.

Massone, M. 1984. Los paraderos tehuelches y prototehuelches en la costa del estrecho de Magallanes. Anales del Instituto de la Patagonia. Serie Ciencias Sociales 15: 27-43 